\title{
Variations
}

Variations

Revue internationale de théorie critique

$16 \mid 2012$

Tahrir is here !

\section{La rage contre le règne de l'argent}

Rage against the rule of money

John Holloway

Traducteur : José Chatroussat

2. OpenEdition

Journals

Édition électronique

URL : http://journals.openedition.org/variations/153

DOI : 10.4000/variations. 153

ISSN : 1968-3960

Éditeur

Les amis de Variations

Édition imprimée

Date de publication : 16 janvier 2012

Référence électronique

John Holloway, «La rage contre le règne de l'argent », Variations [En ligne], 16 | 2012, mis en ligne le 20 février 2012, consulté le 01 mai 2019. URL : http://journals.openedition.org/variations/153 ; DOI :

10.4000 /variations. 153

Ce document a été généré automatiquement le 1 mai 2019.

Les ami•e•s de Variations 


\section{La rage contre le règne de l'argent}

Rage against the rule of money

John Holloway

Traduction : José Chatroussat

\section{NOTE DE L'ÉDITEUR}

Intervention à Istanbul en avril 2011

Première publication sur www.theoriecritique.com, « Tahrir is here ! », 16 janvier 2012,

pp. 18-24

1 La rage. Ce sont des jours de rage. Pas seulement le vendredi mais tous les jours. Il y a la rage en Afrique du Nord et dans le monde arabe bien sûr, mais aussi dans les rues d'Athènes, de Dublin, de Rome, Paris, Madrid, Lisbonne, et il y a une rage profonde qui couve au Mexique.

2 Une époque de crise comme l'époque actuelle est un temps de rage. C'est une époque d'attentes frustrées, d'espoirs frustrés, de vies frustrées. Nous voulons étudier à l'université mais c'est trop cher et il n'y a pas de bourses d'études. Nous avons besoin d'une bonne assurance de santé mais nous n'avons pas l'argent pour la payer. Nous avons besoin d'un logement, nous voyons des logements vides mais ils ne sont pas pour nous. Ou bien encore, tout simplement, des millions et des millions de gens affamés dans le monde peuvent s'écrier: Nous voulons manger; nous voyons que la nourriture existe, qu'il y a largement assez de nourriture pour tout le monde sur cette planète mais qu'il y a quelque chose qui s'interpose entre nous et la nourriture. C'est l'argent ou plutôt le fait que nous n'en avons pas assez.

3 Nous sommes donc en rage. Et nous le sommes d'autant plus que nous ne savons pas quoi faire de notre rage. Nous ne savons pas comment utiliser notre rage pour construire un monde différent. 
4 Nous sommes en rage contre ce qui est évident, contre le gouvernement - contre Erdogan, contre Berlusconi, Papandréou, Kadhafi, Moubarak. Mais nous savons qu'il n'y a aucune réponse à trouver là. Notre colère ne se soulève pas seulement contre les politiciens, mais contre la pauvreté et la frustration que représentent ces politiciens. Nous savons, ou tout du moins ceux d'entre nous qui ont vécu dans des démocraties savent, que la démocratie représentative maintient notre rage emprisonnée. Comme un rat dans un labyrinthe sans issue, nous courons d'un parti à un autre, d'un dirigeant à un autre, mais il n'y a pas moyen d'en sortir, les choses ne vont pas mieux, ne peuvent pas aller mieux, parce que derrière le pouvoir politique s'en tient un autre, un pouvoir plus grand, le pouvoir du capital, le pouvoir de l'argent.

5 Notre rage risque de s'épuiser dans des impasses, ce qui est dangereux parce que nous savons que cela peut facilement se transformer en amertume. Cela devient une rage étouffée, une rage suffocante, une rage sans espoir, une rage qui mène facilement à la violence domestique, au racisme et même au fascisme. Que signifie cette montée de la droite partout dans le monde, si ce n'est l'expression de cette rage sans espoir, sans perspective?

6 Dans cette rage des espoirs détruits, la rage, le plus souvent n'apparaît pas. Tout ce que nous voyons, ce sont les espoirs détruits, la dépression, les antidépresseurs, les drogues. Où la rage se trouve-t-elle, demandent les gens, lorsqu'il semble que le monde se caractérise par l'acceptation, par la soumission aux ordres des riches et des puissants. C'est une rage structurelle, une rage encastrée dans la frustration sur laquelle le monde est construit. C'est une rage qui ne peut pas se manifester mais qui est toujours latente, toujours menaçante, qui demande d'être atteinte. Une rage que ceux d'entre nous qui ont la chance de vivre loin des centres du pouvoir et de l'argent peuvent peut-être discerner plus facilement. Une rage que nous respectons parce que c'est notre rage. Une rage qui est la seule source possible de changement social radical. Et nous qui pensons le changement social, nous devons nous efforcer de la chevaucher, comme si c'était un tigre.

7 La rage, donc, comme point de départ. Mais quoi, ensuite ? Où, ensuite ? Où allons-nous avec notre rage? Notre rage contre le monde qui nous entoure est une rage justifiée parce que le monde est injuste, destructeur et suicidaire. Notre rage est plus que justifiée, elle est juste, elle est une rage de rectitude, une rage de dignité. Une rage qui propulse vers un monde différent, une rage qui ne sera satisfaite que lorsque nous aurons créé un monde qui ne sera pas fondé sur le management de la peur.

8 Nous sommes donc en rage, pas seulement contre les politiciens, pas seulement contre les banquiers, pas seulement contre les riches et les capitalistes: nous sommes en rage contre eux bien sûr mais ils ne sont puissants que dans la mesure où ils servent une plus grande puissance, l'argent. Nous sommes en rage contre le règne de l'argent.

9 Cela ne veut pas dire que nous ne voulons pas d'argent, par nécessité. L'argent est la forme que prend la richesse dans cette société, et en tant que producteurs de cette richesse, nous voulons tous en avoir notre part. Dans la société actuelle, quel que soit le degré d'austérité auquel nous pouvons (ou ne pouvons pas) aimer vivre, nous avons besoin d'argent pour réaliser nos projets. Alors oui, nous voulons de l'argent, pour nousmêmes, pour les universités, pour les écoles et les hôpitaux, pour les jardins et les parcs, etc, pour tous les projets qui s'orientent vers un monde différent. Mais nous ne voulons pas d'un monde qui est gouverné par l'argent. Nous ne voulons pas d'un monde dans 
lequel l'argent est la forme dominante de la cohésion sociale, le médium à travers lequel s'instaurent nos relations sociales.

10 L'argent est séduisant. Il a un visage d'impartialité et d'égalité. Et cependant nous savons qu'il n'en est pas ainsi. L'existence de l'argent comme étant la force dominante de la cohésion sociale a des conséquences désastreuses et peut-être fatales pour l'humanité. L'argent nous prive de la possibilité de notre autodétermination, car l'existence de l'argent comme cohésion sociale signifie que la force qui façonne le développement social est une force que personne ne contrôle. Les gouvernements prétendent contrôler le développement de la société mais encore et encore, il se dégage clairement qu'ils sont soumis au mouvement de l'argent. Ils peuvent toujours essayer de contrôler ce mouvement mais ils ne peuvent y parvenir. L'argent a sa propre dynamique, la dynamique de l'auto-expansion, la dynamique du capital. L'argent s'efforce constamment de s'accroître. Il veut s'engouffrer partout où les possibilités d'expansion sont les plus grandes. En fin de compte l'expansion de l'argent dépend de la production de plus-value, de l'intensification continuelle de l'exploitation et de la course incontrôlable du plus vite, plus vite, plus vite. Le règne de l'argent est le règne du capital. Le règne de l'argent est la soumission du monde à une dynamique que personne ne contrôle, une dynamique qui crée des contrastes énormes entre les riches et les pauvres, qui crée de la violence et des guerres, une dynamique qui détruit les autres formes de vie sur terre, qui détruit les conditions primordiales de l'existence humaine : une dynamique qui n'est pas seulement destructrice mais suicidaire. L'argent est un grand bulldozer qui réduit en miettes le monde autour de nous. L'argent est une force insidieuse qui pénètre toujours davantage d'aspects de nos vies et de nos relations avec les autres gens. Le mouvement de l'argent est le mouvement de la désintégration sociale. L'argent maintient l'ensemble de la société, mais il le fait d'une façon telle qu'il la déchire en lambeaux et la conduit à sa propre destruction. L'argent détruit la communauté humaine.

11 La rage donc, la rage contre le règne de l'argent! Pourtant cela semble si désespéré, si ridicule. La rage contre la police et vous jetez une pierre. La rage contre les banques et vous brisez une vitre. Mais comment êtes-vous en rage contre l'argent? Cela donne l'impression de descendre dans la rue pour cogner dans l'air. Où d'aller au bord de la mer et de frapper l'eau. L'argent semble si naturel. Il semble faire simplement partie de l'atmosphère que nous respirons.

12 Et pourtant il n'en est pas ainsi. En fait nos vies sont une lutte perpétuelle contre la domination de l'argent, une lutte continuelle pour créer des espaces ou des moments que nous protégeons contre les assauts de l'argent. Nous créons des zones interdites à l'argent ; nous plaçons des pancartes qui disent : « Dehors l'argent! Dehors le capital ! Ici, le peuple gouverne! Ici, dans nos rapports avec nos enfants et nos amis, ici dans nos écoles, ici dans nos hôpitaux, il y a une autre dynamique en œuvre. Dehors l'argent! Ici, la loi de la valeur n'a pas sa place, ici nous créons et défendons des valeurs différentes ! Et nous avons de nombreux noms différents pour désigner ces moments et ces espaces; nous les appelons amour ou fraternité ou solidarité de classe ou camaraderie ou confiance ou anti-pouvoir ou communauté ou communisme. Le communisme, compris de cette façon, fait tout simplement partie de la lutte quotidienne : c'est ce qui fait que la création d'une société communiste est une possibilité réelle.

13 L'histoire est une lutte de classe, bien sûr, mais au cours des derniers siècles, la lutte des classes a été centrée sur l'argent : d'un côté la lutte pour imposer l'argent comme le lien omniprésent, s'infiltrant dans tous les recoins de nos vies, et de l'autre la lutte à tous les 
niveaux pour briser la dynamique meurtrière du règne de l'argent (du règne du capital) et pour créer-et-défendre des formes différentes de relations entre les gens autour de nous.

Nous pensions autrefois que nous avions repoussé de manière décisive le règne de l'argent. Ce fut peut-être le grand espoir, la réussite et finalement le mythe du vingtième siècle. Nous pensions que dans une grande partie du monde, suite aux révolutions russe, chinoise et autres, l'argent ne déterminait plus le développement social. Et il n'y avait pas que cela : avec l'Etat providence dans de nombreux pays, le règne de l'argent semblait avoir été refoulé de domaines comme la santé, l'éducation et, jusqu'à un certain point, le logement. Il y avait en cela quelque chose de réel : les grandes luttes du vingtième siècle ont réellement refoulé la domination de l'argent de manières telles que la qualité de la vie en a été changée de façon significative. Pouvoir se rendre chez le médecin sans se soucier de l'argent à payer, pouvoir envoyer ses enfants à l'école sans se soucier du coût des études - ce sont des conquêtes qui ne doivent pas être éliminées, ce sont des conquêtes que nous devons défendre là où elles existent encore.

Cependant le recul du règne de l'argent n'a pas été la rupture de son pouvoir que nous pouvions espérer. Sous les coups massifs de celles et ceux qui luttaient pour un monde différent, l'argent battit en retraite et se ressaisit, rassemblant ses forces en vue d'un nouvel assaut. Cette retraite a pris la forme de l'Etat providence keynésien et des États socialistes. Dans les deux cas, l'Etat joua le rôle-clé. L'Etat sembla fournir l'alternative au règne de l'argent : le règne de l'Etat remplaçait, semblait-il, le règne de l'argent. Et bien sûr, l'Etat était démocratique, soumis au gouvernement du peuple. Mais l'Etat ne peut pas remplacer le règne de l'argent parce qu'il dépend de l'argent pour exister : il dépend de l'argent collecté par les impôts ou par la vente des produits fabriqués par les entreprises d'Etat. Et le règne de l'Etat n'est certainement pas le gouvernement du peuple parce que l'Etat, en tant que forme d'organisation fondée sur l'administration de la société par des fonctionnaires à plein temps, empêche systématiquement de diriger sa propre vie. Autrement dit, une faiblesse résidait au cœur des grandes luttes contre le règne de l'argent: elles étaient focalisées sur la forme d'organisation, l'Etat, qui paraissait être contre le règne de l'argent mais qui, en fait, prolongeait et étendait ce règne. La grande dynamique de la mort n'était pas brisée.

16 Pour le dire en d'autres termes, l'argent est une forme de cohésion sociale, de lien social qui se retourne contre ce que nous faisons, contre la façon dont nous le faisons et contre chaque détail de notre vie. L'argent façonne notre faire mais en même temps, il est produit et reproduit par la manière dont nous faisons les choses que nous faisons. L'argent transforme notre activité, notre faire, en travail ou plus précisément, en travail abstrait et aliéné ; et c'est ce travail abstrait qui produit et reproduit le règne de l'argent. Le règne de l'argent est le règne du travail abstrait: l'un renforce l'autre. La seule manière de briser le règne de l'argent (la dynamique mortifère) est de transformer l'activité sur laquelle il repose, en brisant le travail abstrait et en le remplaçant par un faire différent, une activité concrète autodéterminée. Mais ni les révolutions socialistes, ni l'Etat providence n'ont fait quoi que ce soit pour briser l'emprise du travail. Au contraire, ils ont renforcé énormément la domination du travail sur les formes alternatives d'activité et ils ont ainsi renforcé le règne de l'argent qu'ils s'efforçaient en apparence de miner.

17 Et ainsi, dans la dernière partie du vingtième siècle, l'argent est réapparu dans toute l'arrogance de son pouvoir non brisé. Les grandes révolutions ont été balayées, l'Etat providence est ouvertement attaqué. L'argent revient avec toute sa violence, chassant des 
millions et des millions de paysans de leur terre, transformant les grandes villes du monde en de gigantesques taudis, soumettant toute vie à la règle du profit, mesurant toute chose. On proclame que l'argent est la seule forme de cohésion sociale, la seule manière rationnelle permettant aux gens de s'associer. Inclinez-vous devant le règne de l'argent ! Le travail abstrait est la seule façon de faire, et le travail abstrait est la règle du toujours plus vite. Inclinez-vous devant le règne de l'argent, inclinez-vous devant le règne du travail!

Mais les gens ne s'inclinent pas ou tout du moins ils ne le font pas suffisamment. Nous voulons toujours vivre même si nous n'avons pas un emploi. Nous voulons toujours aimer, et non pas avoir des relations mesurées par l'argent. Nous pouvons travailler plus vite mais nous ne pouvons pas suivre le rythme du toujours plus vite, plus vite, plus vite, exigé par l'argent, par le capital. Et ainsi, comme déjà dans les années 1920, mais de plus en plus à mesure que le siècle avance, la résistance populaire pénètre dans l'argent comme un ver pénètre dans une pomme et se développe en elle. La seule façon dont l'argent/le capital peut se reproduire et maintenir son emprise sur le monde ne peut se faire que sur la base d'une expansion toujours croissante de la dette. Mais cela le rend de plus en plus instable et sujet à des crises périodiques. La période de l'arrogance du capital (le néolibéralisme) a été caractérisée par l'instabilité financière qui a conduit au grand krach de 2008.

La crise financière est la manifestation explosive de l'incapacité de l'argent à fournir la cohésion sociale qu'il prétend fournir. Une très grande partie de la population mondiale est tout simplement laissée en marge par l'argent : elle reçoit très peu d'argent et trouve des manières de survivre (ou pas) sans passer par la forme de l'argent. Dans une crise financière, la faillite de la cohésion explose : de plus en plus de gens sont exclus du circuit de l'argent ou inclus de façon marginale ou précaire. La fragilité du règne de l'argent se révèle. De plus en plus de gens sont forcés, souvent contre leur volonté, de trouver d'autres formes de cohésion sociale, d'autres formes pour se reproduire eux-mêmes. En période de fort chômage, par exemple, et en particulier dans les pays où il y a peu ou pas d'indemnités de chômage, la solidarité de la famille élargie devient cruciale.

Par temps de crise, l'antagonisme entre l'argent et les autres formes de cohésions sociales s'intensifie. Précisément au moment de sa faillite, l'argent (avec le soutien de ses loyaux serviteurs, les politiciens) fait tout ce qui est possible pour maintenir et renforcer son pouvoir : c'est la signification des crises et des interventions de l'Union Européenne et du FMI en Grèce, en Irlande et au Portugal.

21 Et de notre côté ? Il y a la rage et la confusion. Mais aussi bien plus que cela. Le dynamisme contre le règne de l'argent est toujours là ; il est toujours la clé de notre humanité et de notre espoir. Et maintenant il n'est plus focalisé sur l'Etat : l'illusion de l'Etat comme alternative à l'argent a été grandement affaiblie, même si elle est encore présente. Actuellement le mouvement contre le règne de l'argent prend de plus en plus la forme de créations d'espaces interstitiels, d'espaces ou de moments dans lesquels des formes expérimentales de cohésion sociale sont créées sur une base différente, suivant consciemment une logique différente. Ce sont les «zones interdites» que nous avons vues plus haut, les espaces d'amour, de confiance ou de communisme qui sont enracinés dans la vie quotidienne mais qui s'engagent plus dans une attaque ouverte contre le règne de l'argent. Elles peuvent être considérées comme des failles dans la texture de la domination capitaliste, des brèches dans le règne de l'argent, des moments ou des espaces qui avancent contre-et-au-delà de la société existante. 
Ces brèches viennent de différentes directions et souvent elles se rejoignent; ou souvent elles ne le font pas. Elles incluent ces formes de solidarité que les gens ont développées par nécessité, simplement comme une façon de survivre. Elles ont été cruciales par exemple dans les soulèvements urbains des villes d'Amérique Latine lors des vingt dernières années. Elles incluent également (d'une manière contradictoire mais très importante) les luttes pour sauvegarder ce qui reste de la dernière grande mobilisation contre le règne de l'argent, l'éducation gratuite, les soins de santé et les autres services fournis (même si c'est de façon inadéquate ou oppressive) par le biais de l'Etat - voyez par exemple les luttes actuelles contre les coupes dans les dépenses publiques en GrandeBretagne. Et troisièmement, il y a les millions d'initiatives et d'expériences créées consciemment en dehors des structures de l'Etat, les milliers et milliers de révoltes et d'expériences à travers le monde où les gens disent : « Non, nous n'allons pas accepter le règne de l'argent, nous n'allons pas accepter le règne du capital, nous allons faire les choses de façon différente. » Il y a tellement de refus-et-créations, tellement de dignités : parfois grandes, parfois petites, parfois pathétiques, toujours contradictoires. Des occupations de logements, des centres sociaux, des jardins communautaires, des stations de radio alternatives, des mouvements de logiciels gratuits, des rébellions paysannes dans lesquelles le peuple dit: «Assez! Maintenant, c'est le peuple qui va gouverner », des occupations d'usines, des manifestations dans des universités comme celle où les étudiants et les enseignants se sont mis d'accord pour décider de se concentrer sur la seule question scientifique qui nous reste à résoudre, à savoir comment pouvons-nous arrêter la course tête baissée de l'humanité vers son autodestruction? Un monde de refus-et-créations, un monde de dignités. Une politique économique de la résistance, une anti-économie de la rébellion, l'embryon peut-être d'un monde nouveau, un monde de nombreux mondes.

Ces brèches sont nos défenses-et-attaques contre la dynamique meurtrière et suicidaire $\mathrm{du}$ règne de l'argent. Ces brèches sont le seul espoir que l'humanité puisse avoir encore un avenir. Le seul espoir de créer un monde radicalement différent réside dans la création, l'expansion, la multiplication et la confluence de ces brèches, de ces dignités, de ces espaces et moments de refus et de création.

La rage donc, la rage contre le règne de l'argent. Briser les vitres des banques, tirer sur les politiciens, tuer les riches, pendre les banquiers aux lampadaires. Tout cela est certainement très compréhensible. Mais c'est l'argent que nous devons tuer, pas ses serviteurs. Et la seule façon de tuer l'argent est de créer des cohésions sociales différentes, des façons différentes de vivre ensemble, différentes façons de faire les choses. Tuons l'argent, tuons le travail. Ici, maintenant. 


\section{AUTEURS}

\section{JOHN HOLLOWAY}

Zapatiste intergalactique, docteur en sciences politiques de l'université d'Edimbourg, Ecosse, professeur à l'Institut des Sciences Sociales et Humanités Francisco Velez Pliego de l'université autonome de Puebla, Mexique. Auteur de Changer le monde sans prendre le pouvoir (2007) et de Crack Capitalism (Pluto Press, Londres, 2010. Traduction française par José Chatroussat à paraître). 